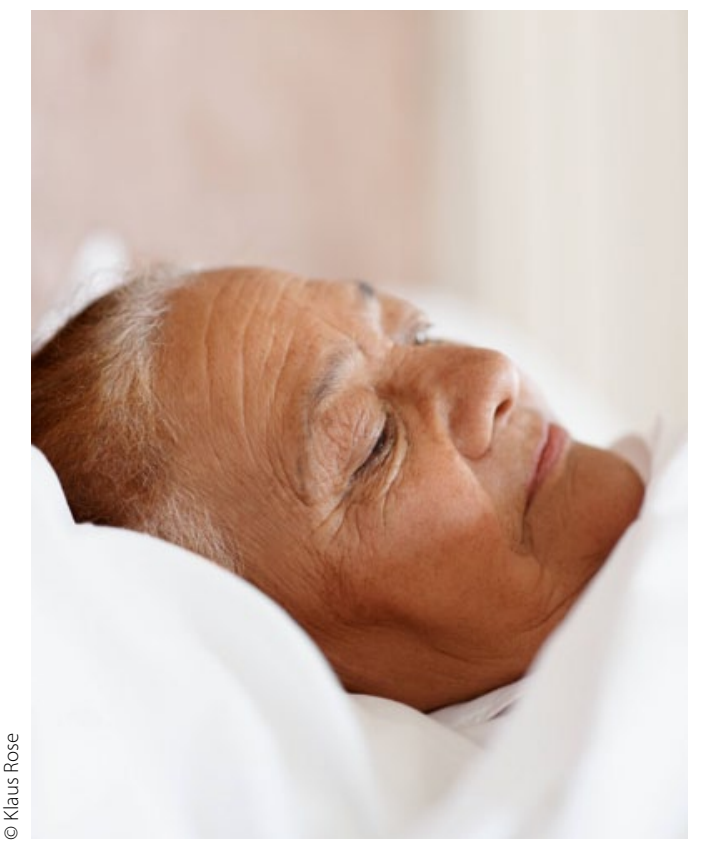

Vergeblich auf den Schlaf warten - gerade für ältere Menschen kann das quälend sein.

\title{
Schlafstörungen
}

\section{Phyto-Arznei mit Vorteilen für Ältere}

\begin{abstract}
- Medikamente, die älteren Menschen ihren Schlaf zurückbringen sollen, haben nicht selten erhebliche Nebenwirkungen, erinnert Bionorica SE. Eine seniorengerechte Auswahl von Medikamenten bietet Ärzten und Apothekern die im Herbst 2010 eingeführte Priscus-Liste. Die Liste umfasst 83 Wirkstoffe, die als potenziell ungeeignet für geriatrische Patienten gelten.

Unter den Sedativa finden sich ältere Antihistaminika (wie Doxylamin und Diphenhydramin) und Benzodiazepine. Alternativ schlägt die Priscus-Liste hoch dosierte Baldrianpräparate zur Therapie bei Insomnie oder eine nicht medikamentöse Therapie der Schlafstörungen (Schlafhygiene) vor.
\end{abstract}

Pflanzliche Arzneien wie Allunapret ${ }^{\oplus}$ seien eine wissenschaftlich gut belegte Alternative zu vielen chemisch-synthetischen Schlafmitteln, teilt das Unternehmen mit. Das Präparat wirke mit einer Kombination methanolischer Trockenextrakte aus Baldrian und Hopfen bei Ein- und Durchschlafstörungen. Die adenosinerge Wirkung von Baldrian erhöhe die Schlafbereitschaft und werde durch die melatonerge Wirkung des Hopfens ergänzt, die den natürlichen SchlafWach-Rhythmus unterstützt. Die Wirksamkeit bei Ein- und Durchschlafstörungen sei in klinischen Studien belegt worden. Die Priscus-Liste im Web: www.priscus.net.

- Red.

Quelle: nach Informationen von Bionorica SE

\section{Koronare Herzkrankheit}

\section{Häufigkeit der Angina pectoris wird unterschätzt}

- Symptomatische Therapieoptionen bei koronarer Herzkrankheit werden nach Einschätzung von Prof. Dr. Gert Richardt, Bad Segeberg, künftig mehr Gewicht erhalten. Er weist darauf hin, dass Angina pectoris nach interventioneller oder medikamentöser Akuttherapie von KHK-Patienten noch relativ häufig vorkomme. So litten z. B. in der COURAGE-Studie fünf Jahre nach Abschluss der Akuttherapie noch bis zu einem Viertel der Patienten an pektanginösen Beschwerden (NEJM 2007; 356:1503-1516).

Derzeit werde der Stellenwert von Nitraten in der kardialen Rehabilitation unterschätzt, meint Richardt. Dies liege zum einen an der verbreiteten Auffassung, dass KHK-Patienten nach abgeschlossener Akutbehandlung angeblich nur selten an Angina pectoris leiden. Zum anderen gebe es eine gewisse Geringschätzung symptomatischer Therapien: „Wenn ein Behandlungs- prinzip nicht die Prognose verbessert, wollen manche davon nichts wissen", kritisiert er. Eine Umfrage in seiner eigenen Klinik ergab, dass nur etwa jeder zehnte KHK-Patient Nitrate (z. B. Nitrolingual ${ }^{\oplus}$ ) erhält. Selbst bei typischen pektanginösen Beschwerden und bei nachgewiesener Ischämie bevorzugten nach Angaben des Kardiologen die meisten Kollegen ein primär invasives Vorgehen.

Laut Nationaler Versorgungsleitlinie "Chronische KHK" sollen Patienten mit stabiler Angina pectoris über ein schnell wirkendes Nitrat zur Kupierung von Anfällen verfügen. Lang wirksame Nitrate verbessern die Symptomatik und Belastungstoleranz bei Angina pectoris.

\section{- Dr. Thomas Meißner}

Quelle: Expertengespräch „Nitroglycerin: Moderne Therapiekonzepte in der Kardiologie", Hamburg, September 2012 (Veranstalter: PohlBoskamp)

\section{Kurz notiert}

Ejaculatio praecox > Mit einer Prävalenz von bis zu $30 \%$ ist die Ejaculatio praecox (EP) weltweit die häufigste sexuelle Dysfunktion bei Männern unter 60 Jahren. Die schlimmste Form ist die Ejaculatio ante portas, bei der Betroffene nicht in der Lage sind, Kinder zu zeugen. „Der vorzeitige Samenerguss hat unglaublich negative Auswirkungen auf die Orgasmusfähigkeit und bedeutet einen enormen Stress für den Patienten und seine Partnerin", erklärte Porst. Der EP liegt eine Störung der serotonergen Transporter zugrunde. Der selektive Serotonin-Wiederaufnahmehemmer (SSRI) Dapoxetin (Priligy ${ }^{\oplus}$ ) flutet schneller an und wird schneller eliminiert als andere SSRI und ist daher nach Thomas Kreutzig, Urologe in Freiburg, als Bedarfsmedikation der EP gut geeignet und derzeit die einzig zugelassene Substanz für diese Indiaktion. Seine Wirksamkeit und Sicherheit ist auch in klinischen Studien dokumentiert.

Berlin-Chemie 